\title{
Numerical Study of Instabilities in Porous Media Using Finite Difference Method
}

\author{
M.S.Prajapati ${ }^{1}$, Priti Tandel ${ }^{2}$, P.H.Bhathawala ${ }^{3}$ \\ ${ }^{1}$ Associate Professor, Muni. Art \& U.B.Science College, Mehsana-384002, Gujarat, India \\ ${ }^{2}$ Department of Mathematics, V.N.S.G.U., Surat-394510, Gujarat, India \\ ${ }^{3}$ UGC Visiting Professor, Dept. Of Mathematics, HNGU Patan, Gujarat, India
}

\begin{abstract}
The present paper deals with the instabilities takes place in two immiscible phase flow through homogeneous porous media. If the flow of two immiscible fluids is considered unidirectional in a large medium, it can be investigated easily. Corresponding to the movement direction, if parameters like pressures, saturations, fluid speeds, etc. changes only in a single space direction then it can be easily investigated. Solution is obtained by finite difference method.
\end{abstract}

Keywords: Instabilities, Porous media, Immiscible, Finite difference method.

\section{Introduction}

The vertical component of the velocities is taken in an account as the porous medium is considered thicker. The region of the medium where saturation of injecting part rises sharply, the interfaces known as front. On the large scale, the front encroachment are called the tongue phenomenon, but on a smaller scale, called fingering. These encroachments satisfied the conditions of stability of instability. Instabilities occur due to the different viscosities of flowing fluids.

Many authors like Scheidegrer, A.E[1,2,3], Verma [4], Coss [5], G. Hu, P.A.

Zegeling [6], Singh T.[7], R.N.Borana, V.H.Pradhan, and M. N. Mehta[8], D.E.HillandJ.Y. Parlange[9] discussed this phenomena in different ways.

Capillary pressure and common mean pressure is taken into consideration to obtain the solution. Fingers are determined by a statistical treatment. The governing equation is solved using finite difference method known as Successive over Relaxation method.

\section{Statement of the problem}

Instead of regular displacement of the whole front, when another fluid of lesser viscosity is injected into a porous medium containing a fluid, protuberance may occur which shoot through the porous medium at relatively great speed. Those protuberances are called fingers and the phenomenon is called fingering. A finite cylindrical piece of homogeneous porous medium of length $\mathrm{L}$ is considered. It is fully saturated with a fluid known as native fluid, which is displaced by injecting the another fluid. This gives rise to protuberance known as fingers. Complete saturation is assumed at the initial boundary.

The cross- sectional area occupied covered by fingers has been is considered. In the statistical treatment of fingers only the average behavior of the two fluids involved is taken into consideration.

\section{Mathematical Formulation of the problem}

According to Darcy's law, the seepage velocity of injected fluid $\left(\mathrm{V}_{\mathrm{i}}\right)$ and native fluid $\left(\mathrm{V}_{\mathrm{n}}\right)$ are :

$$
\begin{aligned}
& \mathrm{V}_{\mathrm{i}}=-\frac{\mathrm{k}_{\mathrm{i}}}{\mu_{\mathrm{i}}} \mathrm{k} \frac{\partial \mathrm{P}_{\mathrm{i}}}{\partial \mathrm{x}} \\
& \mathrm{V}_{\mathrm{n}}=-\frac{\mathrm{k}_{\mathrm{n}}}{\mu_{\mathrm{n}}} \mathrm{k} \frac{\partial \mathrm{P}_{n}}{\partial \mathrm{x}}
\end{aligned}
$$

Here k-permeability of the homogeneous medium, $\mathrm{k}_{\mathrm{i}}, \mathrm{k}_{\mathrm{n}}$ - relative permabilities of injected fluid and native fluid respectively, $\mathrm{P}_{i}, \mathrm{P}_{n}$ - pressures of injected fluid and native fluid respectively and $\mu_{\mathrm{i}}, \mu_{\mathrm{n}}$ - viscosities of injected fluid and native fluid respectively. Again $\mathrm{k}_{\mathrm{i}}$ and $\mathrm{k}_{\mathrm{n}}$ are assumed to be functions of saturations $S_{\mathrm{i}}$ and $\mathrm{S}_{\mathrm{n}}$ respectively.

The continuity equations are:

$$
\begin{gathered}
\varphi \frac{\partial S_{i}}{\partial t}+\frac{\partial V_{i}}{\partial x}=0 \\
\varphi \frac{\partial S_{n}}{\partial t}+\frac{\partial V_{n}}{\partial x}=0
\end{gathered}
$$

Where $\varphi$ is the porosity of the medium.

The porous medium is considered to be fully saturated. 
Therefore from the definition of phase saturation,

$$
\mathrm{S}_{\mathrm{i}}+\mathrm{S}_{\mathrm{n}}=1
$$

The capillary pressure $\left(\mathrm{P}_{\mathrm{c}}\right)$, across their common interfaceis:

$$
\mathrm{P}_{\mathrm{c}}=\mathrm{P}_{\mathrm{n}}-\mathrm{P}_{\mathrm{i}}
$$

Assuming the relationship between the relative permabilities, phase saturation and capillary pressure as follow:

$$
\begin{aligned}
& \mathrm{k}_{\mathrm{i}}=\mathrm{S}_{\mathrm{i}}, \mathrm{k}_{\mathrm{n}}=\mathrm{S}_{\mathrm{n}}=1-\mathrm{S}_{\mathrm{i}} \\
& \mathrm{P}_{\mathrm{c}}=-\beta \mathrm{S}_{\mathrm{i}}
\end{aligned}
$$

Here negative sign shows the direction of saturation of water is opposite to capillary pressure, where $\beta$ is however small.

The pressure of native fluid $\left(\mathrm{P}_{\mathrm{n}}\right)$ can be written as

$$
\begin{aligned}
& \mathrm{P}_{\mathrm{n}}=\frac{1}{2}\left(\mathrm{P}_{\mathrm{n}}+\mathrm{P}_{\mathrm{i}}\right)+\frac{1}{2}\left(\mathrm{P}_{\mathrm{n}}-\mathrm{P}_{\mathrm{i}}\right) \\
& \mathrm{P}_{\mathrm{n}}=\overline{\mathrm{P}}+\frac{\mathrm{P}_{\mathrm{c}}}{2}, \overline{\mathrm{P}}=\frac{\mathrm{P}_{\mathrm{n}}+\mathrm{P}_{\mathrm{i}}}{2}
\end{aligned}
$$

Where $\overline{\mathrm{P}}$ is the mean pressure which is assumed as a constant.

Substituting(1) and (2) into (3) and (4)

$$
\begin{gathered}
\varphi \frac{\partial \mathrm{S}_{\mathrm{i}}}{\partial \mathrm{t}}=\frac{\partial}{\partial \mathrm{x}}\left[\frac{\mathrm{k}_{\mathrm{i}}}{\mu_{\mathrm{i}}} \mathrm{k} \frac{\partial \mathrm{P}_{\mathrm{i}}}{\partial \mathrm{x}}\right] \\
\varphi \frac{\partial \mathrm{S}_{n}}{\partial \mathrm{t}}=\frac{\partial}{\partial \mathrm{x}}\left[\frac{\mathrm{k}_{\mathrm{n}}}{\mu_{\mathrm{n}}} \mathrm{k} \frac{\partial \mathrm{P}_{\mathrm{n}}}{\partial \mathrm{x}}\right]
\end{gathered}
$$

Eliminating $\frac{\partial P_{i}}{\partial x}$ from equations (11) and (6), we get

$$
\varphi \frac{\partial \mathrm{S}_{\mathrm{i}}}{\partial \mathrm{t}}=\frac{\partial}{\partial \mathrm{x}}\left[\frac{\mathrm{k}_{\mathrm{i}}}{\mu_{\mathrm{i}}} \mathrm{k}\left\{\frac{\partial \mathrm{P}_{n}}{\partial \mathrm{x}}-\frac{\partial \mathrm{P}_{\mathrm{c}}}{\partial \mathrm{x}}\right\}\right]
$$

From(5),(11) and (12), we obtain

$$
\frac{\partial}{\partial \mathrm{x}}\left[\left\{\frac{\mathrm{k}_{\mathrm{i}}}{\mu_{\mathrm{i}}}+\frac{\mathrm{k}_{\mathrm{n}}}{\mu_{\mathrm{n}}}\right\} \mathrm{k} \frac{\partial \mathrm{P}_{n}}{\partial \mathrm{x}}-\frac{\mathrm{k}_{\mathrm{i}}}{\mu_{\mathrm{i}}} \mathrm{k} \frac{\partial \mathrm{P}_{\mathrm{c}}}{\partial \mathrm{x}}\right]=0
$$

Integrating equation (13) with respect to $x$, we get

$$
\left[\left\{\frac{\mathrm{k}_{\mathrm{i}}}{\mu_{\mathrm{i}}}+\frac{\mathrm{k}_{\mathrm{n}}}{\mu_{\mathrm{n}}}\right\} \mathrm{k} \frac{\partial \mathrm{P}_{n}}{\partial \mathrm{x}}-\frac{\mathrm{k}_{\mathrm{i}}}{\mu_{\mathrm{i}}} \mathrm{k} \frac{\partial \mathrm{P}_{\mathrm{c}}}{\partial \mathrm{x}}\right]=-\mathrm{c}
$$

Where $\mathrm{c}$ is the constant of integration (negative sign on right hand side is considered for our convenience).

Simplifying equation (14), we get

$$
\frac{\partial \mathrm{P}_{n}}{\partial \mathrm{x}}=-\frac{\mathrm{A}}{\mathrm{k}\left\{\frac{\mathrm{k}_{\mathrm{i}}}{\mu_{\mathrm{i}}}+\frac{\mathrm{k}_{\mathrm{n}}}{\mu_{\mathrm{n}}}\right\}}+\frac{\mathrm{k}_{i}}{\mu_{\mathrm{w}}\left\{\frac{\mathrm{k}_{\mathrm{i}}}{\mu_{\mathrm{i}}}+\frac{\mathrm{k}_{\mathrm{n}}}{\mu_{\mathrm{n}}}\right\}} \frac{\partial \mathrm{P}_{\mathrm{c}}}{\partial \mathrm{x}}
$$

Now substituting equation (15) into equation (12), we have

$$
\begin{gathered}
\varphi \frac{\partial S_{i}}{\partial t}=\frac{\partial}{\partial x}\left[\frac{\mathrm{k}_{\mathrm{i}}}{\mu_{\mathrm{i}}} \mathrm{k}\left\{-\frac{\mathrm{A}}{\mathrm{k}\left\{\frac{\mathrm{k}_{\mathrm{i}}}{\mu_{\mathrm{i}}}+\frac{\mathrm{k}_{\mathrm{n}}}{\mu_{\mathrm{n}}}\right\}}+\frac{\mathrm{k}_{\mathrm{w}}}{\mu_{\mathrm{w}}\left\{\frac{\mathrm{k}_{\mathrm{i}}}{\mu_{\mathrm{i}}}+\frac{\mathrm{k}_{\mathrm{n}}}{\mu_{\mathrm{n}}}\right\}} \frac{\partial \mathrm{P}_{\mathrm{c}}}{\partial \mathrm{x}}-\frac{\partial \mathrm{P}_{\mathrm{c}}}{\partial \mathrm{x}}\right\}\right] \\
\varphi \frac{\partial \mathrm{S}_{\mathrm{i}}}{\partial \mathrm{t}}+\frac{\partial}{\partial \mathrm{x}}\left[\frac{\mathrm{k}_{\mathrm{n}}}{\mu_{\mathrm{n}}} \mathrm{k} \frac{\partial \mathrm{P}_{\mathrm{c}}}{\partial \mathrm{x}} \frac{1}{\left\{1+\frac{\mathrm{k}_{\mathrm{i}} \mathrm{k}_{\mathrm{n}}}{\mu_{\mathrm{i}} \mu_{\mathrm{n}}}\right.}+\frac{\mathrm{A}}{\left\{1+\frac{\mathrm{k}_{\mathrm{i}} \mathrm{k}_{\mathrm{n}}}{\mu_{\mathrm{i}} \mu_{\mathrm{n}}}\right\}}\right]=0
\end{gathered}
$$

Now from (9), $\quad \frac{\partial \mathrm{P}_{n}}{\partial \mathrm{x}}=\frac{1}{2} \frac{\partial \mathrm{P}_{\mathrm{c}}}{\partial \mathrm{x}}$

Substituting this value in (14), we have

$$
\mathrm{A}=\left\{\frac{\mathrm{k}_{\mathrm{i}}}{\mu_{\mathrm{i}}}-\frac{\mathrm{k}_{\mathrm{n}}}{\mu_{\mathrm{n}}}\right\} \frac{\mathrm{k}}{2} \frac{\partial \mathrm{P}_{\mathrm{c}}}{\partial \mathrm{x}}
$$

Substituting the value of A from (17) into (16), we get

$$
\begin{gathered}
\varphi \frac{\partial \mathrm{S}_{\mathrm{i}}}{\partial \mathrm{t}}+\frac{\partial}{\partial \mathrm{x}}\left[\mathrm{k} \frac{\mathrm{k}_{\mathrm{i}}}{2 \mu_{\mathrm{i}}} \frac{\partial \mathrm{P}_{\mathrm{c}}}{\partial \mathrm{x}}\right]=0 \\
\varphi \frac{\partial \mathrm{S}_{\mathrm{i}}}{\partial \mathrm{t}}+\frac{\partial}{\partial \mathrm{x}}\left[\mathrm{k} \frac{\mathrm{k}_{\mathrm{i}}}{2 \mu_{\mathrm{i}}} \frac{\mathrm{dP}_{\mathrm{c}}}{\mathrm{dS}_{\mathrm{i}}} \frac{\partial \mathrm{S}_{\mathrm{i}}}{\partial \mathrm{x}}\right]=0
\end{gathered}
$$

From equations $(7),(8)$ and $(18)$, we have

$$
\varphi \frac{\partial S_{i}}{\partial \mathrm{t}}-\frac{\beta \mathrm{k}}{2} \frac{\mathrm{k}_{\mathrm{i}}}{\mu_{\mathrm{i}}} \frac{\partial^{2} \mathrm{~S}_{\mathrm{i}}}{\partial \mathrm{x}^{2}}=0
$$

which is the governing equation of our problem.

A set of boundary conditions are written as

$$
\begin{gathered}
\mathrm{S}_{\mathrm{i}}(0, \mathrm{t})=\mathrm{s}_{1}, \\
\mathrm{~S}_{\mathrm{i}}(\mathrm{L}, \mathrm{t})=\mathrm{s}_{2} \\
\mathrm{~S}_{\mathrm{i}}(\mathrm{x}, 0)=0,0 \leq \mathrm{x} \leq \mathrm{L}
\end{gathered}
$$

Also we assume that there is no flow across the face $\mathrm{x}=\mathrm{L}$ as the face at $\mathrm{x}=\mathrm{L}$ is assumed to be imperable), that is, 
Let $\quad X=\frac{x}{L}, \quad T=\frac{k_{i}}{2 \mu_{i}} \frac{k}{\varphi L^{2}} t$

From (19), we have

$$
\frac{\partial S_{i}}{\partial T}-\beta \frac{\partial^{2} S_{i}}{\partial X^{2}}=0
$$

with $\mathrm{S}_{\mathrm{i}}(0, \mathrm{~T})=\mathrm{s}_{1}, \mathrm{~S}_{\mathrm{i}}(1, \mathrm{~T})=\mathrm{s}_{2}$

$$
S_{i}(X, 0)=0,0 \leq X \leq 1
$$

IV. Mathematical Solution

Using S.O.R. method [11, 12], we have

Let $\mathrm{r}=\frac{\mathrm{k}}{\mathrm{h}^{2}}$,

$$
s_{i_{m, n+1}}=s_{i_{m, n}}+\frac{\beta k}{2 h^{2}}\left(s_{i_{m}+1, n}-2 s_{i_{m, n}}+s_{i_{m-1, n}}+s_{i_{m}+1, n+1}-2 s_{i_{m, n+1}}+s_{i_{m}-1, n+1}\right)
$$

$$
\begin{aligned}
\lambda_{\mathrm{m}}=\mathrm{s}_{\mathrm{i}_{\mathrm{m}, \mathrm{n}}}+ & \frac{\beta \mathrm{r}}{2}\left(\mathrm{~s}_{\mathrm{i}_{\mathrm{m}+1, \mathrm{n}}}-2 \mathrm{~s}_{\mathrm{i}_{\mathrm{m}, \mathrm{n}}}+\mathrm{s}_{\mathrm{i}_{\mathrm{m}-1, \mathrm{n}}}\right) \mathrm{s}_{\mathrm{i}_{\mathrm{m}, \mathrm{n}+1}} \\
& =(1-\omega) \mathrm{s}_{\mathrm{i}_{\mathrm{m}, \mathrm{n}}}+\omega\left[\frac{\beta \mathrm{r}}{2(1+\beta r)}\left(\mathrm{s}_{\mathrm{i}_{\mathrm{m}+1, \mathrm{n}}}+\mathrm{s}_{\mathrm{i}_{\mathrm{m}-1, \mathrm{n}+1}}\right)+\frac{\lambda_{m}}{(1+\beta r)}\right]
\end{aligned}
$$

Choose $\mathrm{k}=0.1, \mathrm{~h}=0.1, \beta=0.1, \omega=1.47, \mathrm{~s}_{1}=0.75, \mathrm{~s}_{2}=0$

$$
\mathrm{s}_{\mathrm{i}_{\mathrm{m}, \mathrm{n}+1}}=-0.47 \mathrm{~s}_{\mathrm{i}_{\mathrm{m}, \mathrm{n}}}+1.47\left[0.25\left(\mathrm{~s}_{\mathrm{i}_{\mathrm{m}+1, \mathrm{n}}}+\mathrm{s}_{\mathrm{i}_{\mathrm{m}-1, \mathrm{n}+1}}\right)+\frac{\lambda_{m}}{(2)}\right]
$$

Where $\lambda_{\mathrm{m}}=0.5\left(\mathrm{~s}_{\mathrm{i}_{\mathrm{m}+1, \mathrm{n}}}+\mathrm{s}_{\mathrm{i}_{\mathrm{m}-1, \mathrm{n}}}\right)$

V. Table

\begin{tabular}{|l|l|l|l|l|l|}
\hline $\mathrm{T} \rightarrow$ & $\mathrm{T}=0.1$ & $\mathrm{~T}=0.2$ & $\mathrm{~T}=0.3$ & $\mathrm{~T}=0.4$ & $\mathrm{~T}=0.5$ \\
\hline $\mathrm{X} \downarrow$ & \multicolumn{5}{|l|}{$\mathrm{S}_{\mathrm{i}}$} \\
\hline 0 & 0.75 & 0.75 & 0.75 & 0.75 & 0.75 \\
\hline 0.1 & 0.275625 & 0.496156008 & 0.511642798 & 0.560391605 & 0.55734135 \\
\hline 0.2 & 0.101292188 & 0.263382478 & 0.339610503 & 0.366633202 & 0.412415537 \\
\hline 0.3 & 0.037224879 & 0.126577152 & 0.204343785 & 0.236688818 & 0.278114779 \\
\hline 0.4 & 0.013680143 & 0.057462757 & 0.113083915 & 0.140215119 & 0.179800003 \\
\hline 0.5 & 0.005027453 & 0.025140091 & 0.058737247 & 0.076885424 & 0.110651122 \\
\hline 0.6 & 0.001847589 & 0.010717262 & 0.029081976 & 0.039703013 & 0.064650801 \\
\hline 0.7 & 0.000678989 & 0.004481861 & 0.013877096 & 0.019580321 & 0.035991621 \\
\hline 0.8 & 0.000249528 & 0.001846735 & 0.006431706 & 0.009312034 & 0.019168384 \\
\hline 0.9 & $9.17017 \mathrm{E}-05$ & 0.000752047 & 0.00290513 & 0.004248097 & 0.009622856 \\
\hline 1.0 & $3.37004 \mathrm{E}-05$ & 0.000294238 & 0.00120572 & 0.001568582 & 0.004360342 \\
\hline
\end{tabular}

\section{Graphical Representation}

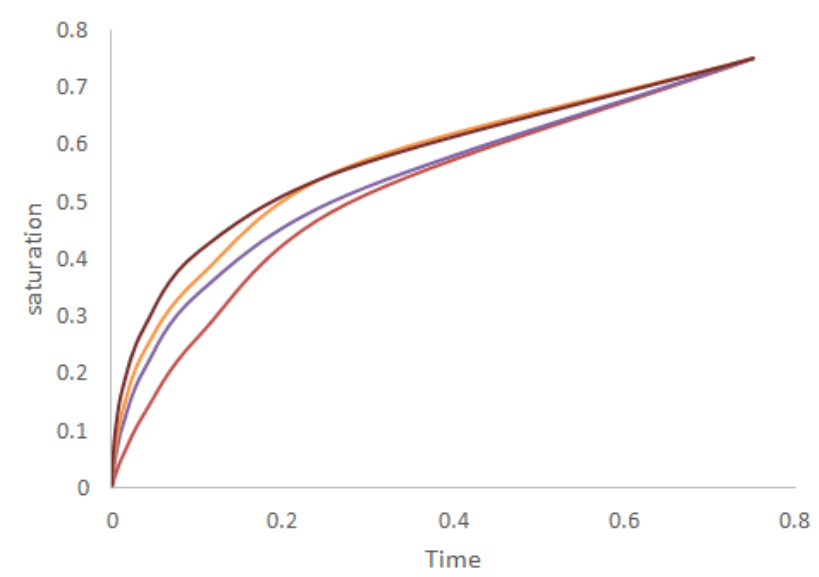




\section{Conclusion}

At the length $\mathrm{X}=0,75 \%$ saturation is considered. It is clear that as time increases the saturation $\left(\mathrm{S}_{\mathrm{i}}\right)$ increases. Also it is clear from the table that as length increases, saturation decreases.

\section{References}

[1]. Scheidegrer ,A.E.(1960): The Physics of Flow through Porous Media , University of Toronto Press, Toronto, 201,216,229

[2]. Scheidegrer,A.E.(1960): Geofishica Pura Appl., 41,47.

[3]. Scheidegger, A.E. and Johnson,E.F. (1961), Canadian Journal Of Physics, 39,329.

[4]. VermaA.P.:Statistical behavior of fingering in displacement process in heterogeneous porous medium with capillary pressure Canadian Journal of Physics, 1969, 47(3): 319-324, 10.1139/p69-042.

[5]. Cosse, R. (1993), Basics of Reservoir Engineering, Editions Technip, Paris.

[6]. G. Hu, P.A. Zegeling: Simulating Finger Phenomena in Porous Media with a Moving Finite Element Method, Journal of Computational Physics (2011).

[7]. Twinkle Singh:The Classical Solution of Burger's Equation Arises into the Fingering Phenomena in Fluid Flow through Homogeneous Porous Media,Applied Mathematics 2011; 1(2): 84-86

[8]. R. N. Borana, V. H. Pradhan, and M. N. Mehta: The Solution of Instability Phenomenon Arising in Homogeneous Porous Media by Crank-Nicolson Finite Difference Method,I.J.of Innovative Research in Science and Technology,Vol.3,Issue-2, 2014,pp.9793-9803.

[9]. D. E.HillandJ.Y. Parlange. Wetting frontinstabilityinlayeredsoils. Soil Sci. Soc. Am. J., 36:697- 702, 1972.

[10]. S.S.Shastry: Introductory Methods Of Numerical Analysis $\left(4^{\text {th }}\right.$ Edition $)$.

[11]. M.K. Jain:Numerical Solution Of Differential Equations $\left(2^{\text {nd }}\right.$ Edition). 